\title{
Inverted J-Shaped Association of High-Sensitivity C-Reactive Protein with the Levels of Serum Uric Acid: Cross-Sectional and Longitudinal Analyses
}

\author{
Xing Zhen Liu $\mathbb{D}^{1, *}$, Ya Jun Tian ${ }^{1} *$, Jie Fan ${ }^{2}$, Lian Yong Liu ${ }^{3}$ \\ 'Department of Health Management, Hangzhou Aeronautical Sanatorium for Special Service of China Air Force, Hangzhou, People's Republic of \\ China; ${ }^{2}$ Department of Traffic Management Engineering, Zhejiang Police College, Hangzhou, People's Republic of China; ${ }^{3}$ Department of \\ Endocrinology, Punan Hospital of Pudong New District, Shanghai, People's Republic of China
}

Correspondence: Jie Fan, Zhejiang Police College, No. 555 Binwen Road, Binjiang District, Hangzhou, People's Republic of China, Tel +86 I3567198867, Fax+8657I3II6I558, Email fanjie@zjjcxy.cn

Lian Yong Liu, Department of Endocrinology, Punan Hospital of Pudong New District, No. 279 Linyi Road, Pudong, Shanghai, 200336, People's Republic of China, Tel +86I3564I44866, Fax +862I58892708, Email chinallu@I63.com

*These authors contributed equally to this work

Experimental and epidemiological studies have demonstrated that hyperuricemia participates in the development of metabolic and cardiovascular diseases by inducing inflammation. ${ }^{1,2}$ In turn, however, the changing pattern of serum uric acid (SUA) levels in the inflammatory environment reported in available literatures is still inconsistent. Several studies had reported that high-sensitivity C-reactive protein (hsCRP), a general marker of systemic inflammation, was positively associated with elevated levels of SUA. ${ }^{3}$ On the contrary, a significant decrease in SUA levels have also been observed during acute gouty and some other inflammatory arthritis. ${ }^{4}$ Thus, we propose a hypothesis that there may be a non-linear relationship between CRP and SUA.

In order to comprehensively evaluate the association of hsCRP with SUA levels, combined cross-sectional and longitudinal study design was used. The cross-sectional and longitudinal clinical data were extracted from our routine health examination database (2013-2021). The inclusion criteria was that those individuals who had complete anthropometric, biochemical, and hsCRP data. Exclusion criteria included acute phase of infectious diseases, autoimmune disease, and the use of UA-lowering drugs and immunomodulators. The use of these data and study protocols were approved by the ethics committee of Punan Hospital of Pudong New District.

Height, weight, waist circumference (WC), and hip circumference were measured by well-trained nurses. Venous blood samples were drawn in the morning at fasting state. Fasting plasma glucose (FPG), SUA, and lipid parameters were measured by a biochemical autoanalyzer (Hitachi 7100). Plasma hsCRP was measured by means of particleenhanced immunonephelometry on biochemical autoanalyzer (Hitachi 7100). Body mass index (BMI) was calculated as weight divided by the square of height; hyperuricemia was defined as SUA $\geq 420 \mu \mathrm{mol} / \mathrm{L}$.

Natural logarithmic transformation of hsCRP was performed to normalize its distribution. Smooth curve fitting was used to visualize the associations of hsCRP with SUA levels. Logistic regression (adjusting for age, gender, smoking, drinking, BMI, WC, FPG, and lipid parameters) and Cox proportional hazards models (adjusting for baseline age, gender, smoking, drinking, BMI, WC, FPG, lipid parameters, and follow-up time) with cubic spline functions were performed to examine the association of hsCRP with hyperuricemia.

A total of 29,926 adults were enrolled in cross-sectional analysis, whose mean age was 46.5 years and $37 \%$ were women. Their mean SUA and hsCRP concentrations were $348.8 \mu \mathrm{mol} / \mathrm{L}$ and $1.68 \mathrm{mg} / \mathrm{L}$, respectively. Another 7178 adults were included in the longitudinal analyses. Their mean baseline age was 48.0 years and $33.8 \%$ were women. The average observational time was 4.1 (1-7) years. Their mean baseline SUA and hsCRP concentrations were $357.9 \mu \mathrm{mol} / \mathrm{L}$ and $1.95 \mathrm{mg} / \mathrm{L}$, respectively.

Received: 18 November 2021 Accepted: 7 January 2022

Published: 16 January 2022 
As shown in Figure 1, the fully adjusted smooth curve fitting presented a non-linear association of hsCRP with SUA and hyperuricemia. It can be seen intuitively that when the Ln (hsCRP) was less than 1.5 (hsCRP $<4.5 \mathrm{mg} / \mathrm{L}$ ), both the SUA levels and hyperuricemia risk elevated with the increase of hsCRP. However, when the Ln (hsCRP) was higher than 1.5 (hsCRP $<4.5 \mathrm{mg} / \mathrm{L}$ ), the SUA levels and hyperuricemia risk began to show a downward trend as hsCRP raised.

$\mathbf{A}_{400-}$

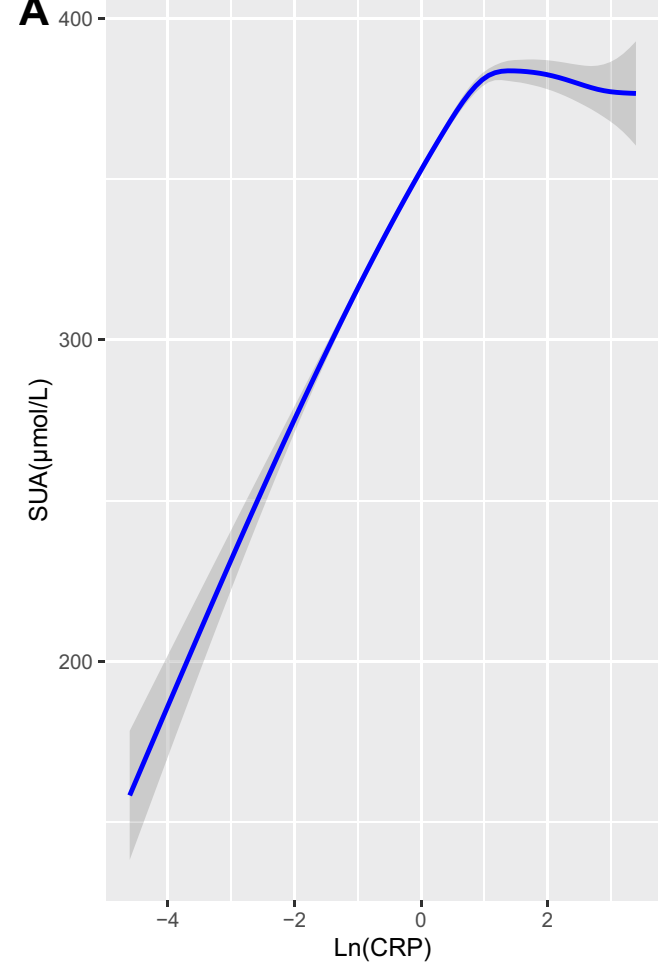

C

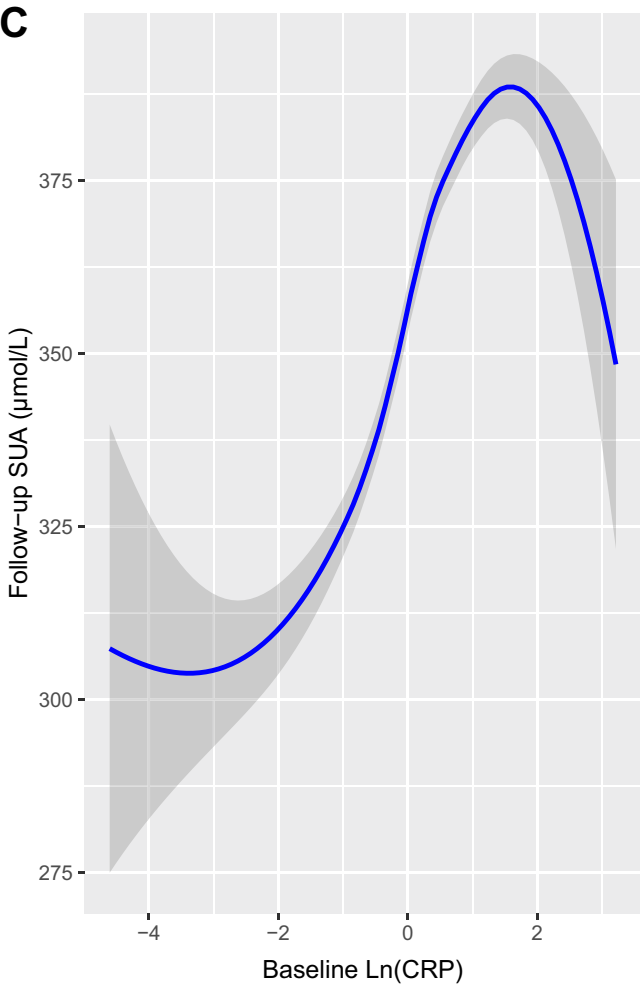

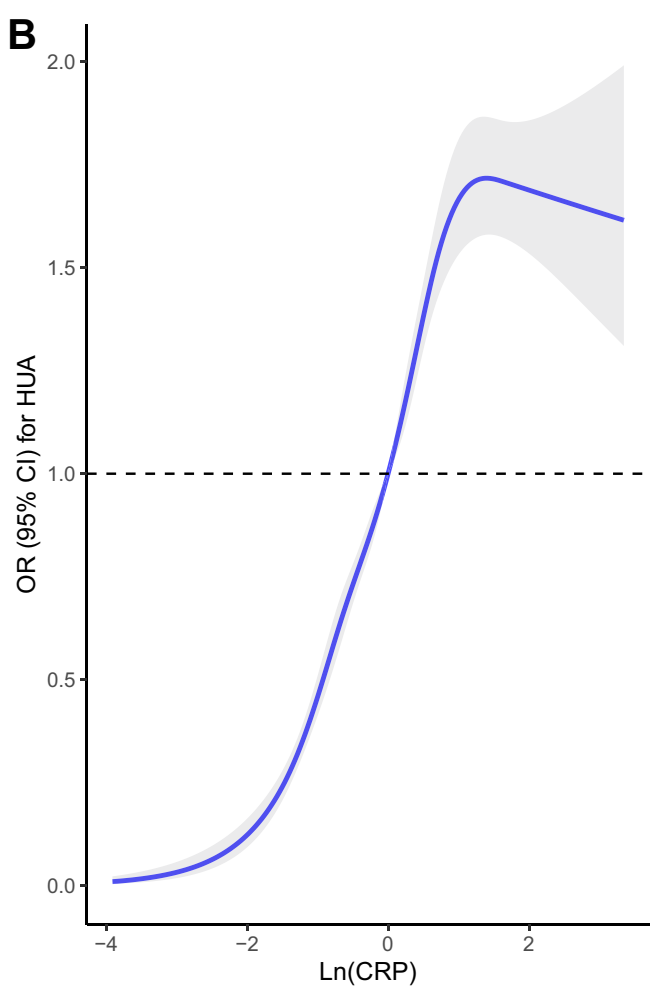

D

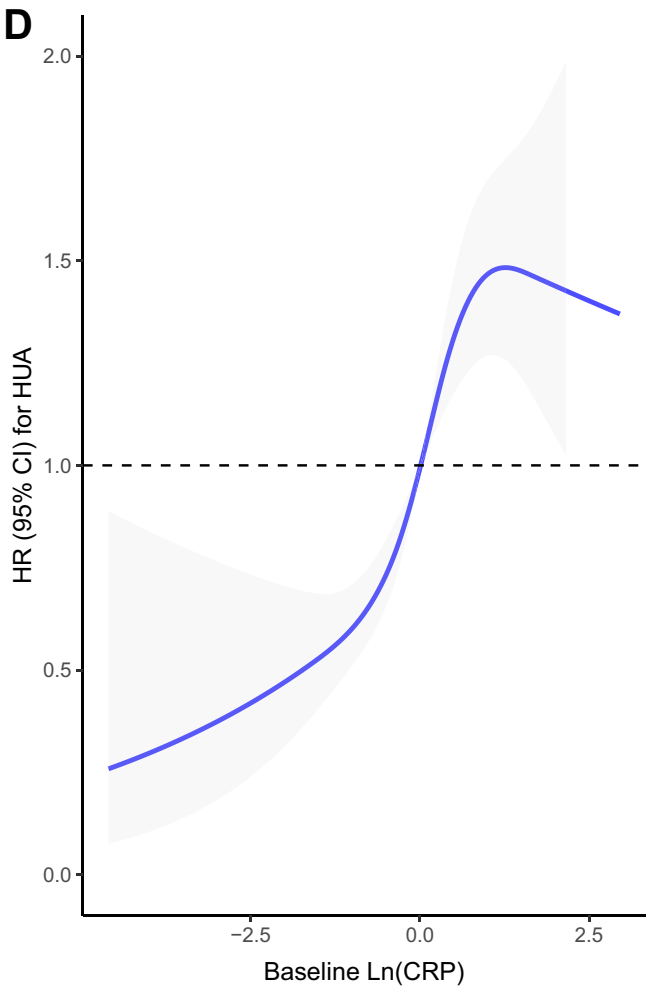

Figure I Cross-sectional (A and B) and longitudinal (C and D) association of hsCRP with SUA and hyperuricaemia.

Abbreviations: SUA, serum uric acid; hsCRP, high-sensitivity C-reactive protein. 
UA is not only a pro-inflammatory agent, but also an antioxidant, which making up about $50 \%$ of the antioxidant capacity present in human biological fluids. Due to the protective effect of UA, theoretically, subclinical chronic lowgrade inflammation may cause an adaptively increase of SUA levels. This may explain the rising part of the fitted curve (hsCRP<4.5mg/L).

When hsCRP continued to rise $(>4.5 \mathrm{mg} / \mathrm{L})$, we found that SUA levels or the risk of hyperuricemia began to drop. This trend of changes in SUA levels can also find good examples in clinical practice. For instance, the levels of serum UA usually decrease during gout flares. ${ }^{4}$ In the situation of acute systemic inflammatory response induced by elective knee or hip surgery, a decrease in SUA concentrations was observed. ${ }^{5}$ Conversely, when systemic inflammation was suppressed, an increase in the levels of SUA in patients with systemic autoimmune rheumatic diseases was also observed. ${ }^{6}$

There are several possible explanations for the decrease in SUA levels in higher inflammatory states. First, the increased excretion of UA in a high-grade inflammation may explain the decrease of SUA levels. Second, the neutralizing effect of UA on free radicals and other oxidative stress factors may consume a part of UA. UA can be non-enzymatically oxidized into allantoin by ROS in spite of the lack of uricase. ${ }^{7}$ Higher levels of allantoin observed in individuals with active rheumatoid arthritis and gout flares are good proof of the above process.

In short, we confirmed the inverted U- or J-shaped association of hsCRP with SUA levels among "apparently healthy" population, both cross-sectionally and longitudinally. Their non-linear relationship can not only help us better understand some interesting clinical phenomena, but also the etiology of hyperuricemia.

\section{Data Sharing Statement}

The datasets used and/or analyzed during the current study are available from the corresponding author on reasonable request.

\section{Ethics Approval and Consent to Participate}

The research protocol was approved by the ethics committee or review committee of the Punan Hospital of Pudong New District.

\section{Consent for Publication}

All authors agree to publish this work.

\section{Acknowledgments}

We would like to thank the colleagues in the Health Management Center, whose long-term work has accumulated a lot of clinical data for us.

\section{Author Contributions}

All authors made a significant contribution to the work reported, whether that is in the conception, study design, execution, acquisition of data, analysis and interpretation, or in all these areas; took part in drafting, revising or critically reviewing the article; gave final approval of the version to be published; have agreed on the journal to which the article has been submitted; and agree to be accountable for all aspects of the work.

\section{Funding}

This study was supported in part by Outstanding Leaders Training Program of Pudong Health Bureau of Shanghai (PWRI2018-02), Special Scientific Research Project on Health Care of People's Liberation Army (14BJZ31), and General Project of Hangzhou Aeronautical Sanatorium for Special Service of China Air Force (20TLMS004).

\section{Disclosure}

The authors report no conflicts of interest. 


\section{References}

1. Joosten LAB, Crişan TO, Bjornstad P, Johnson RJ. Asymptomatic hyperuricaemia: a silent activator of the innate immune system. Nat Rev Rheumatol. 2020;16(2):75-86. doi:10.1038/s41584-019-0334-3

2. Kocak MZ, Aktas G, Erkus E, Sincer I, Atak B, Duman T. Serum uric acid to HDL-cholesterol ratio is a strong predictor of metabolic syndrome in type 2 diabetes mellitus. Rev Assoc Med Bras. 2019;65(1):9-15. doi:10.1590/1806-9282.65.1.9

3. Dai HX, Zhao ZY, Xia Y, Wu QJ, Zhao YH. Higher levels of high-sensitivity c-reactive protein is positively associated with the incidence of hyperuricemia in Chinese population: a report from the China health and retirement longitudinal study. Mediators Inflamm. 2020;2020:3854982. doi: $10.1155 / 2020 / 3854982$

4. Urano W, Yamanaka H, Tsutani H, et al. The inflammatory process in the mechanism of decreased serum uric acid concentrations during acute gouty arthritis. J Rheumatol. 2002;29(9):1950-1953.

5. Waldron JL, Ashby HL, Razavi C, et al. The effect of the systemic inflammatory response, as provoked by elective orthopaedic surgery, on serum uric acid in patients without gout: a prospective study. Rheumatology. 2013;52(4):676-678. doi:10.1093/rheumatology/kes327

6. Pietsch DEW, Kubler P, Robinson PC. The effect of reducing systemic inflammation on serum urate. Rheumatology. 2020;59(10):3108-3109. doi:10.1093/rheumatology/keaa085

7. Kaur H, Halliwell B. Action of biologically-relevant oxidizing species upon uric acid. Identification of uric acid oxidation products. Chem Biol Interact. 1990;73(2-3):235-247. doi:10.1016/0009-2797(90)90006-9

Journal of Inflammation Research

\section{Publish your work in this journal}

The Journal of Inflammation Research is an international, peer-reviewed open-access journal that welcomes laboratory and clinical findings on the molecular basis, cell biology and pharmacology of inflammation including original research, reviews, symposium reports, hypothesis formation and commentaries on: acute/chronic inflammation; mediators of inflammation; cellular processes; molecular mechanisms; pharmacology and novel anti-inflammatory drugs; clinical conditions involving inflammation. The manuscript management system is completely online and includes a very quick and fair peer-review system. Visit http://www.dovepress.com/testimonials.php to read real quotes from published authors.

Submit your manuscript here: https://www.dovepress.com/journal-of-inflammation-research-journal 